\title{
Análisis de técnicas mnemónicas de la entrevista cognitiva
}

Marisol Elizalde Monjardin,, ${ }^{1,2}$, Robyn Holliday ${ }^{1}$ Heather Flowe ${ }^{1}$

\section{Introducción}

Durante mucho tiempo, los policías, detectives y demás implicados en hacer valer la ley han entrevistado a testigos. Esto ha ayudado a obtener la información que requieren, pero hace más de dos décadas que se creó una nueva forma de entrevistar que permite a los testigos recordar más información. Ésta fue nombrada Entrevista Cognitiva (EC).

La EC fue desarrollada en la década de 1980 por Geiselman y Fisher con la finalidad de ayudar a los testigos a recordar el evento con más claridad y detalle. El objetivo de este tipo de entrevista es recuperar la mayor cantidad de detalles del suceso sin aumentar la cantidad de información incorrecta recordada (Geiselman, Fisher, MacKinnon y Holland, 1986). Originalmente, la Ec se compone de cuatro técnicas mnemónicas diseñadas para auxiliar al testigo a recordar un evento.

2 Universidad Autónoma de Sinaloa. 
1. Reconstrucción del contexto (RC): esta técnica incita al testigo a realizar una reconstrucción mental del contexto físico (aspecto del lugar) y personal (aspectos internos del participante) en el momento que sucedió el evento.

2. Reportar todo (RT): requiere una narración completa del evento que atestiguó.

3. Cambio de orden (co): el evento es relatado en orden distinto a como se presenció, comúnmente comenzando por el final y narrado hacia el comienzo.

4. Cambio de perspectiva (CP): el testigo describe el evento desde una perspectiva distinta a la suya.

En 1992, Fisher y Geiselman agregaron una fase de preguntas después de las técnicas mnemónicas y otras mejoras a la EC. Éstas incluyen técnicas de entrevista, como permitir que el participante avance a su propio ritmo, una interacción del entrevistador flexible, preguntas abiertas en su mayoría, establecimiento de rapport, reconstrucción mental guiada y mejoras en la comunicación entre el entrevistador y el entrevistado. Algunos estudios afirman que la efectividad de la EC se debe a la combinación de sus técnicas mnemónicas y que no hay técnica en particular que sea responsable por su eficacia y superioridad sobre otro tipo de entrevistas (Fisher y Geiselman, 1988; Geiselman et al., 1986; Milne y Bull, 2002).

Estudios diversos han demostrado que la combinación de técnicas mnemónicas, como RC y RT, aunada a las técnicas de entrevista mejoradas de la EC son más eficientes que una entrevista estructurada y de menor duración que una Ec completa (Bensi, Nori, Gambetti y Giusberti, 2011; Davis, McMahon y Greenwood, 2005; Milne y Bull, 2002). Además, una EC omitiendo las técnicas mnemónicas de co y cP más una instrucción adicional de reportar el evento, ha demostrado ser superior que una entrevista estructurada (Davis et al., 2005; Ginet y Verkampt, 2007); sin embargo, se ha encontrado que la técnica mnemónica co mejora el reporte de detalles correctos inconsistentes con el contexto (Ginet, Py y Colomb, 2014) y tiene un nivel de precisión mayor que otro tipo de entrevistas (Davis et al., 2005). 
La necesidad de continuar examinando los beneficios de las técnicas mnemónicas de la EC sigue siendo imperativa. Por lo tanto, el objetivo principal de este estudio es examinar la efectividad de técnicas mnemónicas de la EC.

\section{Método}

\section{Participantes}

Un total de 60 estudiantes universitarios de la licenciatura en Psicología de la UAS ( $M$ edad $=19.37, S D=2.80$ ) participaron en el experimento.

\section{Materiales}

Se empleó un video de breve duración con una connotación negativa como el evento a recordar. El video muestra una escena de un asalto a una joven, donde el sujeto logra quitarle la bolsa y huir del lugar. Cuatro distintos tipos de entrevistas se emplearon: 1) una EC completa, 2) RC + RT, 3) RT + co y 4) RT + CP.

\section{Procedimiento}

El experimento se llevó a cabo en dos fases, de manera individual y en dos laboratorios distintos. Durante la primera fase los participantes miraban el video y contestaban un cuestionario acerca de las emociones que sintieron al ver el video (dicho cuestionario sirvió como distracción para encubrir el propósito real del experimento). La segunda fase se llevó a cabo después de 24 horas en un laboratorio distinto al de la primera fase. Durante esta fase los participantes fueron entrevistados empleando uno de los tipos de entrevistas de manera aleatoria. La primera autora realizó todas las entrevistas. Las instrucciones fueron leídas de manera literal. Todas las entrevistas contaron con establecimiento de rapport, instrucciones generales, fase de recuerdo libre y fase de preguntas. 


\section{Resultados}

Una serie de 4 (tipo de entrevista: $\mathrm{EC}, \mathrm{RC}+\mathrm{RT}$, RT + CO, RT + CP) ANCOVAs con número de detalles correctos, incorrectos y confabulados reportados como variables dependientes y duración de la entrevista como covariable se llevaron a cabo. No se encontraron diferencias significativas para la cantidad de detalles correctos y confabulados recordados; sin embargo, se encontró una diferencia significativa en el número de detalles incorrectos reportados, $F(3,55)=2.90, p<.05, \eta_{p}^{2}=.136$. Una comparación por pares empleando el método de Bonferroni en spss demostró que los participantes de la entrevista RT + co reportaron un mayor número de detalles incorrectos (con índices de ajuste de $M=$ 18.68, SE $=1.60,95 \% \mathrm{Cl}[15.47,21.90])$ que los participantes de la entrevista RT + CP $(M=12.28, S E=1.65,95 \%$ Cl $[8.98,15.58])$. Ninguna otra comparación resultó significativa $(p>.05)$.

Para comprender de dónde surgen los detalles reportados por los participantes, se realizó una serie de 4 (tipo de entrevista) Ancovas de los detalles de acuerdo a la fase en la que fueron reportados (fase de recuerdo libre y fase de preguntas) con duración recuerdo libre como covariable. Se encontraron diferencias significativas por tipo de entrevista en el número de detalles correctos reportados, $F(3,55)=3.45, p<.05, \eta^{2}{ }_{p}$ $=$.158. Una comparación por pares empleando el método de Bonferroni demostró que los participantes de la entrevista RT + co reportaron más detalles correctos $(M=50$. 76, $S E=2.86$, $95 \% \mathrm{Cl}[45.02,56.50])$ que los participantes de la EC $(M=34.44$, $S E=3.80,95 \% \mathrm{Cl}[26.83,42.05])$, ninguna otra comparación resultó significativa. Además, se encontraron diferencias significativas en el número de detalles incorrectos reportados, $F(3$, $55)=2.96, p<.05, \eta_{p}^{2}=.139$. Una comparación por pares empleando el método de Bonferroni demostró que los participantes de la entrevista RT $+\mathrm{CO}(M=6.89, \mathrm{SE}=4.16,95 \% \mathrm{Cl}[5.16$, 8.63]) reportaron un mayor número de detalles incorrectos que los participantes de la entrevista RT $+C P(M=3.68$, $S E=0.89$, $95 \%$ c $[1.90,5.46])$. No se encontró diferencia significativa en el número de detalles confabulados. 
Se realizó una serie de 4 (tipo de entrevista) Ancovas con número de detalles correctos, incorrectos y confabulados reportados en la fase de preguntas como variable dependiente y duración de fase de preguntas como covariable. Los resultados no encontraron diferencias significativas por tipo de entrevista en el número de detalles reportados.

Los resultados encontrados indican que la entrevista RT + co ayuda a los participantes a reportar un mayor número de detalles correctos en la fase de recuerdo libre que otras modificaciones de la EC (e.g., RC + RT, RT + CP Y EC); sin embargo, es precisamente la entrevista RT + co la que más reporta detalles incorrectos.

\section{Discusión}

Inicialmente no se encontraron diferencias en el número de detalles correctos reportados por tipo de entrevista, esto concuerda con Milne y Bull (2002) y Geiselman et al. (1986), quienes sugieren que no es una técnica mnemónica en particular la que hace la Ec efectiva, sino la combinación de técnicas. Estos resultados se deben interpretar con cautela, ya que los detalles reportados corresponden a la entrevista en su totalidad (fase de recuerdo libre y fase de preguntas). Por ello, el análisis de detalles por fase fue llevado a cabo.

En la fase de recuerdo libre los participantes reportaron un mayor número de detalles correctos en la entrevista RT + co. Esto concuerda con un estudio llevado a cabo por Bensi et al. (2011), donde se encontró que la técnica de co aumenta el número de detalles correctos recordados durante la fase de recuerdo libre; sin embargo, Davis et al. (2005) encontraron que un intento adicional de RT produce un número mayor de detalles correctos que la técnica de co.

Es importante señalar que se encontró que es precisamente la entrevista RT + co la que tiene un número mayor de reporte de detalles incorrectos. Una manera de contrarrestar este efecto es por medio de instrucciones específicas donde se recuerda al testigo/participante que no debe inventar información. Al igual 
que puede emplear frases del tipo "no sé" o "no recuerdo" cuando sea necesario en lugar de intentar adivinar la información (Memon, Meissner y Fraser, 2010).

Al igual que en estudios previos (e.g. Ginet et al., 2014), durante la fase de preguntas no se encontraron diferencias significativas entre el número de detalles reportados. Estos resultados son esperados, ya que las entrevistas no difieren durante dicha fase. Cuando se compara una EC o alguna modificación de ella contra una entrevista estructurada se espera encontrar resultados significativos entre los detalles reportados, ya que las instrucciones difieren entre tipos de entrevistas (Bensi et al., 2011; Davis et al., 2005; Holliday et al., 2011; Memon, Wark, Bull y Koehnken, 1997; Wright y Holliday, 2007).

En conclusión, este estudio demuestra que la combinación de técnicas mnemónicas de RT + co conduce a un mayor número de detalles correctos reportados en la fase de recuerdo libre que otras combinaciones de técnicas mnemónicas. Incluso, la entrevista de RT + co ayuda a reportar más detalles correctos que una Ec completa cuando la duración de la entrevista es controlada como una covariable. No obstante, se debe proceder con precaución, ya que es precisamente la entrevista RT + co la que mayor número de detalles incorrectos reporta. Esto nos lleva a sugerir el uso de una combinación de técnicas mnemónicas de la Ec, como lo es la RT + co en situaciones donde el tiempo para realizar la entrevista es reducido.

\section{Referencias}

Bensi, L., Nori, R., Gambetti, E. y Giusberti, F. (2011). The Enhanced Cognitive Interview: A Study on the Efficacy of Shortened Variants and Single Techniques. Journal of Cognitive Psychology, 23(3), 311-321. doi:10.1080/20445 911.2011.497485.

Davis, M.R., McMahon, M. y Greenwood, K.M. (2005). The Efficacy of Mnemonic Components of the Cognitive Interview: Towards a Shortened Variant for Time-Critical Investigations. 
Applied Cognitive Psychology, 19(1), 75-93. doi:10.1002/ acp.1048.

Fisher, R.P. y Geiselman, R.E. (1988). Enhancing Eyewitness Memory with the Cognitive Interview. En R.N. Sykes (Ed.), Practical Aspects of Memory: Current Research and Issues, Vol. 1: Memory in Everyday Life. (pp. 34-39). Oxford England: John Wiley \& Sons.

Fisher, R. P. y Geiselman, R.E. (1992). Memory-Enhancing Techniques for Investigative Interviewing: The Cognitive Interview. Springfield, IL England: Charles C. Thomas, Publisher.

Geiselman, R.E., Fisher, R.P., MacKinnon, D. P. y Holland, H. L. (1986). Enhancement of Eyewitness Memory with the Cognitive Interview. The American Journal of Psychology, 99(3), pp. 385-401.

Ginet, M., Py, J. y Colomb, C. (2014). The Differential Effectiveness of the Cognitive Interview Instructions for Enhancing Witnesses' Memory of a Familiar Event. Swiss Journal of Psychology, 73(1), 25-34. doi:10.1024/1421-0185/a000118. Ginet, M. y Verkampt, F. (2007). The Cognitive Interview: Is its Benefit Affected by the Level of Witness Emotion? Memory, 15(4), 450-464. doi:10.1080/09658210601092670.

Holliday, R.E., Humphries, J.E., Milne, R., Memon, A., Houlder, L., Lyons, A. y Bull, R. (2011). Reducing Misinformation Effects in Older Adults with Cognitive Interview Mnemonics. Psychology and Aging, doi:10.1037/a0022031.

Memon, A., Meissner, C.A. y Fraser, J. (2010). The Cognitive Interview: A Meta-Analytic Review and Study Space Analysis of the Past 25 Years. Psychology, Public Policy, and Law, 16(4), 340-372. doi:10.1037/a0020518.

Memon, A., Wark, L., Bull, R. y Koehnken, G. (1997). Isolating the Effects of the Cognitive Interview Techniques. British Journal of Psychology, 88(2), 179-197. doi:10.1111/j.20448295.1997.tb02629.x.

Milne, R. y Bull, R. (2002). Back to Basics: A componential Analysis of the Original Cognitive Interview Mnemonics with Three Age Groups. Applied Cognitive Psychology, 16(7), 743-753. doi:10.1002/acp.825. 
Wright, A.M. y Holliday, R.E. (2007). Interviewing Cognitively Impaired Older Adults: How Useful is a Cognitive Interview? Memory, 15(1), 17-33. doi:10.1080/09658210601047351. 